\title{
TV/Series
}

3 | 2013

Écho et reprise dans les séries télévisées (I) : Reprise et intermédialité

\section{Glee et la reprise jubilatoire}

Virginie Marcucci

\section{(2) OpenEdition \\ Journals}

Édition électronique

URL : http://journals.openedition.org/tvseries/723

DOI : 10.4000/tvseries.723

ISSN : 2266-0909

Éditeur

GRIC - Groupe de recherche Identités et Cultures

Référence électronique

Virginie Marcucci, « Glee et la reprise jubilatoire », TV/Series [En ligne], 3 | 2013, mis en ligne le 15 septembre 2013, consulté le 20 avril 2019. URL : http://journals.openedition.org/tvseries/723 ; DOI : 10.4000/tvseries.723

\section{(c) $\oplus \Theta \Theta$}

TV/Series est mis à disposition selon les termes de la licence Creative Commons Attribution - Pas d'Utilisation Commerciale - Pas de Modification 4.0 International. 


\section{Glee et la reprise jubilatoire}

Virginie MARCUCCI

Cet article s’intéresse aux différents niveaux auxquels la reprise fonctionne dans la série Glee, diffusée par la FOX depuis 2009. En effet, la reprise y est un concept polyvalent servant aussi bien à construire un univers rigide et normé qu'à le saper et à le déstabiliser. Dans un premier temps, on remarquera que la reprise est de type générique et marque Glee comme une teen series, avec son cortège de situations éculées et de personnages parfois un peu attendus. À cette reprise générique s'ajoute une reprise de clichés et de stéréotypes appliqués aux comportements (notamment genrés) des protagonistes, permettant de construire des personnages masculins et féminins conformes aux attentes de la société, extra-diégétique (composée des téléspectateurs) ou intra-diégétique (composée des personnages). Pourtant, le lieu de la diégèse - le "glee club » auquel appartiennent les héros - introduit grâce à la performance théâtrale et musicale un nouveau type de reprise, qui joue un rôle différent et floute les contours pourtant si bien dessinés au premier abord des identités de genre des personnages. C'est ici le rôle queer de la reprise qui nous intéressera, au sens où ce terme est entendu par Judith Butler, c'est-à-dire en ce qu'elle permet de tordre et de courber les lignes apparemment droites et rectilignes des comportements de genre attachés à chacun des deux sexes biologiques. Enfin, on verra que cette subversion, si elle bouleverse et remet en jeu l'ordre apparemment naturel des choses, est également le prérequis à la construction d'un nouvel ordre (fût-il caractérisé par la mouvance et la fluidité). En effet, elle permet in fine un positionnement très progressiste de la série et de son propos : une fois les attributs de genre montrés comme n'étant pas fermement, ni naturellement, attachés à un sexe ou à l'autre, il est facile d'en faire de même pour la sexualité et l'attente souvent systématique d'une hétérosexualité (ou hétéronormativité).

$\mathrm{L}$ a série Glee (FOX, 2009-), dont le titre signifie en anglais " jubilation », «joie », " allégresse », est le fruit de la collaboration du prolifique duo formé par Ryan Murphy et Brad Falchuk, à qui l'on doit aussi la récente American Horror Story (FX, 2011-). Ryan Murphy a également créé Popular (The WB, 1999-2001), Nip/Tuck (FX, 2003-2010) et The New Normal (NBC, 2012-), tandis que Brad Falchuk a produit nombre d'épisodes de Nip/Tuck. S'il fait bien référence au sentiment que fait naître la série chez les téléspectateurs amateurs de comédies musicales, le titre "Glee » est aussi une allusion aux « glee clubs », expression désignant, aux ÉtatsUnis, des chorales essentiellement scolaires. Sans grande surprise eu égard à son titre, cette série diffusée sur la chaîne Fox $^{1}$ depuis quatre saisons s'intéresse à la reprise en main par Will Schuester (professeur d'espagnol au moment où commence la série) de la chorale du lycée McKinley, situé dans la petite ville de Lima, Ohio. Il est aidé dans sa tâche par la conseillère d'orientation du lycée, Emma Pillsbury, et connaît une adversaire redoutable en la personne de Sue Sylvester,

${ }^{1}$ Notons dès à présent qu'à la différence d'HBO ou de Showtime, par exemple, la Fox est un network, ce qui laisse moins de marge de manœuvre aux concepteurs de séries qui y sont diffusées, puisqu'elles sont soumises aux réglementations de la FCC. 
l'entraîneuse de l'équipe de cheerleaders $^{2}$, qui règne en maître depuis des années sur les compétitions nationales.

Les élèves qui appartiennent à ce « glee club » depuis ses débuts sont Artie Abrams, Rachel Berry, Mercedes Jones, Tina Cohen-Chang et Kurt Hummel. Ils sont rapidement rejoints par les très populaires Quinn Fabray, Santana Lopez, Brittany Pierce (toutes cheerleaders), Finn Hudson et Noah Puckerman (membres phare de l'équipe de football américain). Puis, au cours des saisons, arriveront Blaine Anderson, Sam Evans ou encore Mike Chang. Le simple choix de ces élèves pourrait à lui seul justifier une étude sur le thème de la reprise. En effet, ces élèves, et notamment les tout premiers membres du « glee club », font partie d'une minorité : Artie Abrams est paraplégique et se déplace en fauteuil roulant, Rachel Berry est élevée par deux hommes homosexuels, Mercedes Jones est afro-américaine et d'un gabarit nettement supérieur à ceux des canons actuels (et plus encore télévisuels), Tina Cohen-Chang est américaine d'origine asiatique et Kurt Hummel est homosexuel. La série joue en permanence de la reprise des clichés associés à chacune de ces minorités, notamment par l'entremise de Sue Sylvester, qui donne à chacun d'eux des surnoms très politiquement incorrects. Ainsi elle appelle Artie, Kurt, Mike, Tina, Mercedes et Matt (jeune afro-américain présent dans la première saison) : "Wheels, Gay Kid, Asian, other Asian, Aretha and Shaft » (1.7), réduisant chacun au stéréotype, au cliché ou à la différence qui le caractérise : elle fait référence à Artie par le terme de " Roues » (celles de son fauteuil roulant), à Kurt par celui de "gamin homosexuel ", à Mike par celui d' " Asiatique » (son origine ethnique), à Tina par celui d'« Autre Asiatique ». Elle met enfin l'accent sur le fait que Mercedes et Matt sont afro-américains en donnant à l'une le nom d'Aretha (pour Aretha Franklin, grande chanteuse afro-américaine) et à l'autre celui de Shaft (héros afro-américain de films policiers des années 70). Comme l'explique Marguerite Chabrol, cela est fait de manière si ouverte et exagérée que la série montre ainsi le jeu entre sa reprise des clichés et le peu de crédit qui leur est associé, permettant alors à cette reprise de les déconstruire et de les mettre à distance au lieu de s'en faire le simple relais :

La série est hyper consciente de ses procédés, soulignés dans un métadiscours constant, comme le montre par exemple le personnage de Kristin Chenoweth découvrant la chorale assemblée qu'elle compare à une publicité Benetton (I-5)3.

${ }^{2}$ Si le terme de cheerleaders est souvent traduit par « pom-pom girls », ces deux termes ne sont pas tout à fait équivalents en réalité : les équipes de cheerleaders sont également composées de garçons et leur activité principale ne se borne pas à faire la claque pendant les matchs de l'équipe qu'ils soutiennent, mais consiste bien à se livrer à des acrobaties dignes de gymnastes. On conservera le terme de cheerleaders tout au long de cet article. 3 Marguerite Chabrol, «Glee: Theatricality!", Positif, n607, Septembre 2011, p. 105 [p.103-105]. Kristin Chenoweth est une actrice américaine, connue entre autres pour ses prestations dans certaines comédies musicales américaines (Wicked par exemple). L'actrice 
Ce n'est cependant pas cette reprise-là qui nous intéresse ici. Ce concept semble particulièrement pertinent sur un plan générique car Glee est constitué de la reprise de thèmes typiques de la série adolescente ( «teen series »), mais également parce que sa raison d'être est la reprise de numéros musicaux, qu'ils soient des tubes plus ou moins contemporains ou des grands classiques de Broadway (ce qui permet alors un enchâssement de la comédie musicale au cour de la comédie musicale). Pourtant, tout n'est pas si simple: Glee, c'est l'histoire de David contre Goliath. Cette petite chorale doit se battre pour exister face à l'équipe de cheerleaders, à qui le succès permet de monopoliser tous les crédits du lycée. Quant aux membres du « glee club », ce sont majoritairement, au départ, ces élèves un peu marginaux qui traversent les couloirs du lycée en espérant éviter les brimades de leurs camarades populaires et tyranniques.

Il s'agira alors d'étudier comment, dans un premier temps, la reprise des situations de la série adolescente permet la création d'une norme de genre fictionnel mais également, à l'intérieur de la diégèse, d'un lieu dans lequel existent de puissantes règles régissant les comportements sexuels et genrés des personnages. Cependant, il faudra, dans un deuxième temps, se pencher sur l'ambiguïté du rôle de cette reprise qui, notamment lorsqu'elle se double de la reprise des numéros musicaux, permet de placer la « performance » et le camp 4 au cœur de ce monde intra-diégétique apparemment très lisse et au fonctionnement rigide, subvertissant alors toutes les normes qui semblaient sous-tendre la série - preuve de la tension productive au cœur de la reprise telle qu'elle est utilisée dans Glee.

\section{La reprise pour construire un cadre}

Les premières minutes du pilote reprennent nombre de codes du film ou de la série pour adolescents dont le lieu de la diégèse serait la « high school » américaine. L'épisode s'ouvre en effet sur l'équipe de

\footnotetext{
joue dans quelques épisodes de Glee le rôle d’April Rhodes, gloire passée du " glee club » du lycée retrouvée par hasard par Will Schuester, son camarade de classe à l'époque.

4 Dans cet article, on entendra le terme de " performance» dans son sens anglais de « représentation, spectacle », et plus particulièrement tel qu'il est utilisé dans la théorie queer et notamment chez Judith Butler. Tout étant construit et rien n'étant naturel, elle considère que le genre est " performatif », c'est-à-dire n'existe que sous la forme d'une "performance » : c'est agir, se présenter comme une femme (ou un homme) qui fait qu'on est de genre féminin (ou masculin), pas un caryotype XX ou XY. Ainsi, adopter des comportements de genre caractéristiques d'un autre sexe que son sexe biologique est possible et permet (par la performativité du genre, sa performance) de se présenter comme ayant un genre différent, même temporairement, de celui auquel nous rattacherait notre sexe biologique. Le « camp », que l'on définira plus précisément dans la suite de cet article (en nous appuyant notamment sur le recueil que lui dédie Fabio Cleto), peut être rapidement défini comme un courant ou une sensibilité apportant plus de crédit à l'artificiel qu'au naturel, qu'il remet ainsi en question.
} 
cheerleaders, composée de jeunes gens beaux et très en forme physiquement 5 , s'entraînant sur une musique forte, au rythme enlevé, sous les ordres d'une entraîneuse tyrannique et impitoyable. Ce passage est immédiatement suivi de l'arrivée d'un professeur manifestement fauché (en témoigne l'état de la voiture qu'il gare dans le parking du lycée), réclamant un devoir en retard à un joueur de football américain, qui - comme de bien entendu - est très occupé avec ses coéquipiers à jeter dans la benne à ordures du lycée un élève apparaissant comme plutôt précieux : il demande aux footballeurs de le laisser enlever sa veste de marque avant de finir avec les ordures ${ }^{6}$. Un peu plus tard dans cette introduction aux codes du lycée, on voit dans le gymnase un groupe de cheerleaders (parmi elles, Quinn Fabray et Santana Lopez) qui regardent des vidéos tournées par Rachel Berry se mettant en scène en train de chanter. Leur visionnage est actif puisqu'il se double de l'écriture de commentaires désobligeants à propos de sa prestation sur le réseau social où ces vidéos ont été postées. Ainsi est montrée à deux reprises la violence ordinaire exercée à l'encontre de ceux qui sont un tant soit peu différents7. Les attentes génériques des téléspectateurs sont donc comblées par la réitération d'un certain nombre de topoï associés au type de série qu'est Glee. Ces débuts peuvent faire penser à d'autres teen series telles Beverly Hills 90210 (FOX, 1990-2000), My So-Called Life (ABC, 1994-1995), Gossip Girl (The CW, 2007-2012), The O.C (FOX, 2003-2007), ou encore One Tree Hill (The WB, 2003-2006 puis The CW 2006-2012).

Cependant, la reprise joue un autre rôle : lorsque c'est celle des clichés et des situations liés à chaque genre (au sens de construction sociale des attributs et comportements attachés à chaque sexe biologique), elle permet la construction non plus d'un horizon d'attente pour les téléspectateurs mais de cadres a priori très rigides dans lesquels les personnages peuvent, voire doivent, évoluer. La première matérialisation de cela est à trouver dans les équipes, constituées d'un côté par les athlètes de sexe masculin (les « jocks », le plus souvent, ici, les joueurs de football américain) et de l'autre par les athlètes de sexe féminin (les cheerleaders). Chaque sexe a son équipe ${ }^{8}$ et, au début en

5 On notera également que l'uniforme de l'équipe comporte de grandes similitudes avec ceux qui sont portés par l'équipe des Toros dans le premier opus (Bring It On, réal. Peyton Reed, 2000) de la franchise de films de cheerleaders Bring It On : Bring It On Again (réal. Damon Santostefano, 2004), Bring It On: All or Nothing (réal. Steve Rash, 2006), Bring It On: In It to Win It (réal. Steve Rash, 2007), Bring It On: Fight to the Finish (réal. Billie Woodruf, 2009).

${ }^{6}$ Ce sont respectivement les personnages de Will Schuester, Finn Hudson et Kurt Hummel qui sont présentés.

7 Cette violence est même montrée trois fois puisque, juste avant ce passage, on voit un membre de l'équipe de football américain (Noah Puckerman) lancer un «slushie » (une sorte de granité sucré) au visage de Rachel.

8 Cette idée d'équipe donnera lieu au fil du temps à des jeux de mots explicites sur l'expression anglaise « to play for a team » permettant de faire des références plus ou moins 
tout cas, ces filles sont hétérosexuelles et ont des relations avec ces garçons, également hétérosexuels. Par exemple, le footballeur Noah Puckerman a une relation avec la cheerleader Santana Lopez tandis que Finn Hudson a une relation très sérieuse (et censément chaste) avec Quinn Fabray. Ces deux derniers personnages sont l'illustration la plus parfaite, quand la série commence, de l'adjectif « straight », que l'on utilisera ici, dans la droite lignée de Judith Butler mais également de Samuel Chambers ${ }^{9}$, pour qualifier tout comportement obéissant à certaines normes, qu'elles soient de genre, de sexualité ou plus généralement sociales. Quinn Fabray répond aux canons classiques de beauté, elle est hétérosexuelle, présidente du club de chasteté et capitaine de l'équipe de cheerleaders (donc extrêmement populaire). Elle est en couple avec Finn Hudson, qui est hétérosexuel, beau d'une manière très académique et d'une gentillesse à toute épreuve. Son rôle de quarterback, poste le plus prestigieux au football américain, le rend très populaire également. Ce garçon et cette fille se comportent conformément à ce qui est attendu d'eux par la société au sens large (celle - intra-diégétique - composée des personnages de la série, mais également celle - extra-diégétique - composée des téléspectateurs) compte tenu de leur sexe biologique, à la fois dans les activités extrascolaires qui sont les leurs (attributs de genre) et dans leur sexualité.

Cette construction "straight » de la série en termes de genre et de sexualité est également marquée par une opposition très rigide et à peine métaphorique entre les espaces du lycée : d'un côté, le terrain de football et les vestiaires des garçons, de l'autre, le terrain ou le gymnase où s'entraînent les cheerleaders. Les couloirs constituent l'un des rares espaces mixtes : la « royauté du lycée ${ }^{10}$ » s'y pavane en uniforme de cheerleader ou en portant la « letter jacket », signe de l'appartenance à l'équipe de football, par exemple, tandis que les souffre-douleur sont remis à leur place par diverses brimades et petites tortures du quotidien. Dans Glee, ce sont les "slushies ${ }^{11}$ » qui sont jetés au visage des élèves martyrisés, ces derniers se retrouvant alors couverts d'un liquide glacé, sucré et très coloré. Ce «bizutage » permanent est devenu la signature visuelle de la série et permet de comprendre la

masquées à la sexualité des personnages. Ainsi dans 2.2 Dave Karofsky et Azimio prennent la « letter jacket » de Finn en disant: «This jacket belongs to people who are actually on the team. But you know what? We're going to restyle it to represent the obvious duality in your sexuality. » (une « letter jacket » est une veste portée aux États-Unis par les membres d'une équipe de sport au lycée ou à la faculté, elle tire son nom de la lettre qui y est cousue, symbole de l'engagement et du succès sportif de son propriétaire).

9 Samuel A. Chambers, The Queer Politics of Television, Londres, New-York, IB Tauris, 2009.

${ }^{10}$ Nous employons le terme à dessein compte tenu de la tradition dans les lycées américains (et passage obligé de toute fiction se déroulant dans une " high school ») d'élire le roi et la reine de la promotion lors du bal de fin d'année.

${ }^{11}$ Les « slushies » sont des boissons glacées sucrées assez proches d’un granité. 
hiérarchie du lycée et sa réorganisation au fil des saisons et des années ${ }^{12}$

L'univers du lycée McKinley est ainsi très réglementé et ceux dont le comportement s'éloigne un tant soit peu du chemin rectiligne qui y prévaut - celui de la norme - sont rappelés à l'ordre, avec violence parfois. On pense à Kurt, dont la différence, vestimentaire pour commencer, lui vaut d'être régulièrement jeté dans la benne à ordures, ou à Artie, qui reçoit un "slushie " (2.11) pour la simple raison qu'il est, selon ceux qui le tourmentent, « a glee loser » : la scène ressemble à une exécution en bonne et due forme même s'il s'agit d'une exécution symbolique et sociale. L'image, violente, nous montre Artie Abrams dans son fauteuil roulant, entouré par certains membres de l'équipe de football américain portant leur veste et tenant chacun deux slushies de couleur rouge sang qu'ils jettent tous au même moment sur Artie. On le voit ensuite dans son fauteuil, recouvert de cette boisson rouge et encerclé par ses agresseurs (voir figure 1). La cruauté du geste et la couleur du liquide pourraient presque rappeler l'humiliation de Carrie dans le film du même nom (réal. Brian de Palma, 1976) lorsque des camarades mal intentionnés renversent du sang d'animal sur elle lors du bal du lycée.

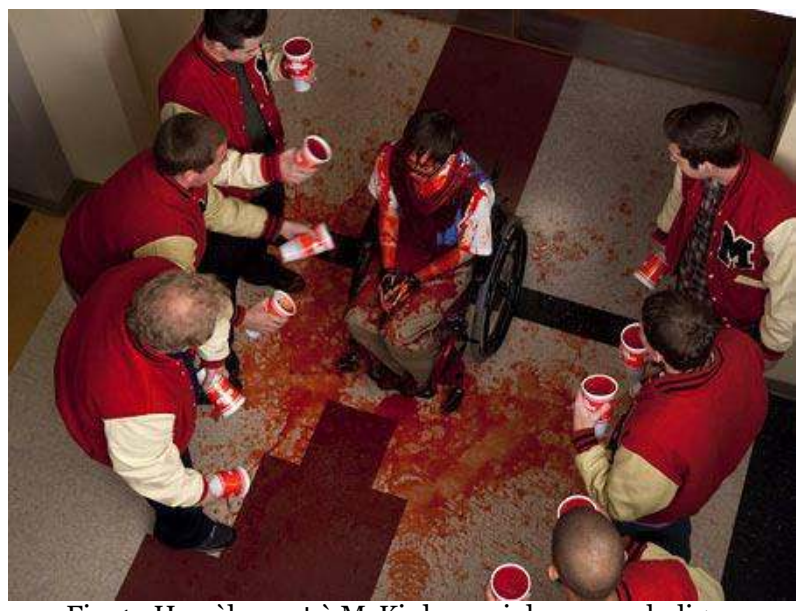

Fig. 1 : Harcèlement à McKinley - violence symbolique $\mathrm{du}$ « slushie » (2.11)

Cette violence, pas toujours symbolique, semble particulièrement s'exercer à l'encontre des garçons : ainsi, dans 1.1,

${ }^{12}$ Ainsi quand certains membres de l'équipe de football ou des cheerleaders choisissent de faire partie du « glee club », ils deviennent à leur tour la cible des «slushies ». 
Ken Tanaka, l'entraîneur de l'équipe de football américain, prédit à Will Schuester les plus grandes difficultés à convaincre ses joueurs de rejoindre son «glee club » en lui expliquant qu'ils ont déjà rasé les sourcils d'un des leurs parce qu'il avait regardé Grey's Anatomy. Grey's Anatomy (ABC, 2005-) est une série médicale dont le fort intérêt pour la vie amoureuse de ses protagonistes la rapproche des codes du soap opera. Ces affinités génériques semblent plutôt la destiner à un public féminin : la regarder correspondrait donc à un comportement de genre féminin, puni par les autres membres de l'équipe désireux de s'assurer que leur camarade se comporte bien en " homme».

Le « glee club » semble en effet entrer en contradiction avec la masculinité et ses attributs tels qu'ils sont habituellement définis : au lycée McKinley, le plus important de ces attributs est l'hétérosexualité mais on peut également citer, plus classiquement, la confiance en soi issue de la force (mentale ou physique) et du pouvoir. Lorsque Will Schuester se rend dans les vestiaires des footballeurs afin de recruter de nouveaux membres pour son " glee club ", les seuls mots ou faux noms inscrits par les joueurs sur l'affiche laissée dans les vestiaires ( « gaylord wiener », " penis », " butt lunch »13) font tous référence soit au sexe masculin soit à l'homosexualité. Cela montre bien à quel point le projet même du « glee club » semble menacer leur masculinité et, par là-même, leur hétérosexualité ${ }^{14}$. De la même manière, Azimio et Dave Karofsky, deux membres de l'équipe de football, expliquent à Finn (dans les toilettes des garçons) :

How many times do we have to go through this? You being a jock and being in this glee club does not make you versatile. It makes you bisexual. [...] Get out of my bathroom. You girls y'all belong across the hall, Glee boy. (1.20)

Cette citation explicite le raisonnement des deux footballeurs : le «glee club» est le royaume de la féminité, tandis que l'équipe de football est celui de la masculinité. Appartenir aux deux n'est pas un signe de polyvalence ou d'adaptabilité (« versatile ») mais celui d'une

\footnotetext{
13 " gaylord wiener » est la combinaison d'un mot signifiant littéralement " seigneur homosexuel " et de " wiener ", terme argotique faisant référence au sexe masculin. Le dernier terme « butt lunch » désigne une pratique sexuelle exclusivement homosexuelle.

14 Contrairement à ce que cette réaction des jeunes sportifs pourrait laisser penser, ce n'est pas simplement le fait de chanter qui menace la masculinité et l'hétérosexualité de ceux qui le font : dans « Accafellas » (1.3), Will, accompagné de collègues (notamment Ken Tanaka) et de certains élèves (Finn Hudson et Noah Puckerman), monte un groupe de chant appelé les «Accafellas " dont les performances semblent au contraire asseoir la virilité de ses chanteurs. Les autres noms envisagés pour leur ensemble étaient « Testostertones » ou "Crescendudes », autant de noms établissant un lien fort entre la musique et le chant d'un côté, et la virilité et la masculinité de l'autre. C'est le chant choral empruntant essentiellement à des chansons de comédies musicales et accompagné de danse (donc, tel qu'il est pratiqué dans le " glee club ») qui semble déviriliser l'activité et émasculer ceux qui y participent.
} 
sexualité de l'entre-deux, ni hétérosexuelle, ni homosexuelle. On remarque également l'équation tendant à faire de "glee » un préfixe démasculinisant, comme si « Glee boy » était un équivalent de " girl » (utilisé dans cette même phrase) ${ }^{15}$.

On le voit, les adolescents eux-mêmes sont de puissants relais de la norme : quand les coéquipiers de Finn Hudson, leur quarterback, comprennent qu'il a manqué leur entraînement afin d'accompagner le " glee club » pour une sortie, il subit une exécution au paintball sur le terrain de football, exécution symbolique certes, mais réellement douloureuse, accompagnée par ces mots de Noah Puckerman : "You broke the rules and for that you must be punished. » (1.1). Cette régulation des comportements continue dans un épisode ultérieur, lorsque Dave Karofsky et Azimio expliquent à Finn Hudson (dans les vestiaires) qu'ils le martyrisent parce qu'il est trop proche à leur goût $\mathrm{du}$ " glee club » (1.12) : " All right, you know the system's put in place to keep order round here ». L'ordre auquel il est fait référence est bien celui d'un certain type de masculinité et de l'hétéronormativité.

D'autres relais de la norme existent au lycée McKinley : parmi eux, les entraîneurs Sue Sylvester et Ken Tanaka, à la tête des équipes de cheerleaders et de football américain. Dès le premier épisode, Sue Sylvester met en mots le code rigide de fonctionnement du lycée en expliquant à Will Schuester :

Okay, so what you're doing now is blurring the lines. High school is a caste system. Kids fall into certain slots. Your jocks and your popular kids up in the penthouse. The invisibles and the kids playing live-action out in the forest ${ }^{16}$ : bottom floor (1.1).

L'image, celle d'une rectitude horizontale, marque bien l'apparente imperméabilité entre les différents groupes évoluant côte à côte (ou, selon ce modèle, les uns au-dessus des autres) au sein du même lycée. Ken Tanaka explique à Finn Hudson quelque chose d'assez similaire lorsqu'il lui dit dans le premier épisode : "You're the quarterback, you make your decision: you're a football player or you're a singer. » Il va même symboliquement plus loin lorsque, dans l'épisode 1.6, il dit à ses joueurs : « Know your routes, know your assignments. » Le conseil est

15 Dans son article « Masculinity as Homophobia », Michael S. Kimmel fait référence à cette manière classique de construire la masculinité - celle qui conduit le petit garçon ou, plus tard, l'homme à se comporter de manière à ne pas être perçu comme une chochotte ("a sissy »). Il écrit: "The fear of being seen as a sissy dominates the cultural definitions of manhood. [...] As adolescents, we learn that our peers are a kind of gender police constantly threatening to unmask us as feminine, as sissies. " in Reconstructing Gender: A Multicultural Anthology, éd. Estelle Disch, Boston, McGraw Hill, 2008, p. 104 [p. 103109].

16 " the kids playing live-action in the forest » est une référence aux adolescents qui se déguisent en trolls, elfes, magiciens etc. selon leurs goûts et qui, ainsi vêtus, laissent libre cours à leur passion pour les jeux de rôles. Ces sessions se passent généralement dans un environnement naturel, comme les forêts. Inutile de dire que ces joueurs sont moins considérés encore que ceux qui s’adonnent aux simples jeux de rôles. 
évidemment un conseil sportif : chaque joueur doit connaître sa place dans l'équipe et ce qu'il doit faire une fois que l'action est lancée sur le terrain. Cependant, compte tenu de tout ce qui vient d'être noté, on peut comprendre que ce conseil montre la nécessité, dans l'ordre de la masculinité, de connaître son itinéraire, de ne pas dévier de sa trajectoire ( « know your routes» vient rappeler le caractère «straight » souhaité pour le comportement de ces adolescents) et de respecter sa place, son affectation ( « know your assignments ») : quand on est génétiquement et biologiquement un homme, il y a des choses qu'on ne fait pas car elles déborderaient du cadre strict de la masculinité telle qu'elle est classiquement entendue.

La série propose donc un univers très réglementé et rigide. Pourtant, c'est justement cette rigidité et ces fréquents rappels à l'ordre qui nous font toucher du doigt la fragilité (et l'arbitraire ?) de la norme. En effet, si cette dernière a tant besoin d'être réaffirmée, c'est parce qu'elle est en danger, tandis qu'une norme puissante fonctionne de manière invisible et tacite. Voici ce qu'écrit Samuel Chambers à propos de l'univers de Desperate Housewives, mais qui s'applique également à celui de Glee :

[T]he show tries so hard to uphold straightness, that it betrays itself. The reason that Wisteria Lane proves something less than straight lies in the very desperation to remain straight. Often the characters on the show work so hard to preserve normality that in the process they reveal the workings of gender and sex norms. [...] In general, to reveal the norm may be to subvert it since norms work best when they are never exposed. In other words, the optimal operation of the norm is an invisible operation. Once norms reach the point that they require significant shoring up, then they have been significantly weakened ${ }^{17}$.

La reprise rigide d'un fonctionnement très binaire (populaire ou non, féminin ou masculin, hétérosexuel ou homosexuel, sportif ou chanteur), royaume du « ou » exclusif, permet en fait à la subversion " queer ${ }^{18}$ » de faire son œuvre en minant et sapant ces normes, non pas en recréant un nouvel ordre, mais en déstabilisant celui qui existe pour mieux le remanier :

Particularly when it comes to the idea of challenging norms, subversion is best thought as a practice that works from inside the terms of the norms. This means that subversion must operate from below. Norms of gender and sexuality cannot easily be overthrown from the outside, but they can be overturned from the inside. Here we have a theory of

\footnotetext{
${ }_{17}$ Chambers, p. 121.

${ }^{18}$ Le terme de " queer », dont l'étymologie le rapprocherait du sens de " tordu, courbé », est utilisé par J. Butler notamment pour faire référence à la manière dont on peut courber/tordre l'apparente rectitude d'un certain nombre de normes (et, en premier lieu, de genre ou de sexualité). Cette opération, selon J. Butler, est rendue effective par la prolifération de combinaisons possibles entre sexe/genre/sexualité permettant de montrer, par exemple, que l'équivalence XY/homme/hétérosexuel n'a rien de naturel et qu'elle est construite culturellement et socialement.
} 
subversion that remains fully inside the terms of culture (norms are cultural artefacts), working with those terms so as to rework them. The subversion of law (and of behaviours, norms, traditions, and practices) operates inside the law (see Butler 1999: 119). This conceptualization of subversion calls up the Latin etymology of the word, subvertere-to turn from below ${ }^{19}$

Plusieurs manières de subvertir les normes existent mais c'est la façon dont ces questions ont été théorisées par Judith Butler qui nous paraît particulièrement féconde pour cette analyse menée sur Glee, car la subversion causée par la prolifération des identités de genre et des combinaisons possibles des trois variables sexe/identités de genre/sexualité est omniprésente au sein du « glee club », permettant alors de tordre, de rendre courbe tout ce qui était si rectiligne et droit (« straight ») à première vue :

No longer believed as an interior "truth" of dispositions and identity, sex will be shown to be a performatively enacted signification (and hence not "to be"), one that, released from its naturalized interiority and surface, can occasion the parodic proliferation and subversive play of gendered meanings. This text continues, then, as an effort to think through the possibility of subverting and displacing those naturalized and reified notions of gender that support masculine hegemony and heterosexist power, to make gender trouble, not through the strategies that figure a utopian beyond, but through the mobilization, subversive confusion, and proliferation of precisely those constitutive categories that seek to keep gender in its place as the foundational illusions of identity ${ }^{20}$.

Ce qui nous intéresse dans cette citation est la manière dont le performatif et la performance au sens queer sont des outils de déstabilisation et de subversion des identités de genre : en faisant proliférer et en multipliant ces identités (une femme pourra ainsi adopter tour à tour des attributs de genre masculin ou féminin), il peut être démontré que le système sur lequel repose une partie de notre société n'est ni naturel ni fixe mais bel et bien construit. Le " glee club » est un parfait écrin pour une telle entreprise en raison de la théâtralité qui est au cœur de son fonctionnement (permettant de passer de la performance musicale à la performance queer) mais également car la reprise de certains numéros musicaux ou de certains artistes permet de rendre particulièrement tangible ce « trouble dans le genre ».

19 Chambers, p. 107

${ }^{20}$ Judith Butler, Gender Trouble, New York, Londres, Routledge, 1990, p. 46, nos italiques. 


\section{La reprise qui subvertit}

Le "glee club » est un des rares lieux mixtes du lycée où se retrouvent dans un premier temps ceux qui sont quelque peu singuliers, et donc à la marge, mais qui attire également quelques-uns des lycéens les plus populaires, qu'ils soient de sexe masculin ou féminin, cheerleaders ou "jocks ». Tous interagissent alors dans le même espace, ce qui permet d'introduire des nuances inconnues dans un monde resté jusque-là fortement contrasté. Notons, pour commencer, le nom de la chorale du lycée McKinley: «New Directions ». Peut-être peut-on y voir de manière liminaire que la déconstruction et la subversion à l'œuvre dans le "glee club » vont permettre d'ouvrir un nouveau chemin, d'aller dans de nouvelles directions et surtout de s'éloigner de l'itinéraire tout tracé qui semblait être le destin de chacun.

Le «glee club» est par essence le lieu de la performance (musicale et théâtrale dans un premier temps), ce qui en fait le lieu d'un enchâssement des performances permettant une mise en évidence de l'aspect performatif de ce qu'on prend en général pour un fait de la nature. Les téléspectateurs sont en effet face à une série télévisée (premier niveau de performance), dans laquelle les personnages se livrent à des performances sous forme de mises en scène de numéros musicaux (deuxième niveau de la performance) tout en jouant également avec leurs attributs de genre et leur sexualité (ce qui fait émerger un troisième niveau de performance possible). Nombreuses sont les fois où les personnages prononcent des phrases telles que "Performing is my life. » (Rachel Berry dans 2.1), «I want to be the spectacle. » (Mercedes dans 3.1) et mettent en valeur lors de leur spectacle de chant cette performance initiale qu'est le " genre ».

On peut rapprocher cela du concept de théâtralité, tellement central au travail de la mise en scène du chant choral qu'il est le titre d'un des épisodes de la première saison. Dans cet épisode, " Theatricality », consacré aux chansons et apparitions scéniques de Lady Gaga, il apparaît clairement, nous allons le voir plus bas, que le déguisement ne vient pas se surajouter à une éventuelle essence préexistante mais que le déguisement EST l'essence, comme l'a écrit Judith Butler à propos de la pratique des drag queens :

The notion of gender parody defended here does not assume that there is an original which such parodic identities imitate. Indeed the parody is of the very notion of an original. [...] This perpetual displacement constitutes a fluidity of identities that suggests an openness to resignification and recontextualization; parodic proliferation deprives 
hegemonic culture and its critics of the claim to naturalized or essentialist gender identities ${ }^{21}$.

Ce lien entre le «glee club» et les problématiques du déguisement et de l'imitation commence à être tissé lors d'épisodes très populaires centrés sur la reprise de chansons d'icônes de la communauté homosexuelle masculine et de divas queers et camps. Ainsi, dans « The Power of Madonna » (1.15), le " glee club » ainsi que l'équipe de cheerleaders de Sue Sylvester trouvent leur inspiration dans l'œuvre de la chanteuse ${ }^{22}$. Pour commencer, l'importance de Madonna dans la culture populaire est affirmée ${ }^{23}$. De plus, divers moments chantés reprennent et mettent en avant tous les avatars de la persona de l'artiste, depuis ses tous premiers clips jusqu'à son style caractéristique de l'album Ray of Light, en passant par les périodes où elle s'inspirait de Marilyn ou d'Evita. L'épisode "Brittany vs Britney » (2.2) est consacré, comme son titre le laisse deviner, aux plus grands tubes de Britney Spears. L'épisode repose sur des séances de rêves/hallucinations vécues par quelques élèves du « glee club » alors qu'ils sont sous anesthésie chez le dentiste. Ainsi, Brittany apparaît dans une version de "Slave $4 \mathrm{U}$ ", Santana ainsi que Brittany rêvent qu'elles chantent «Me against the Music»24, Rachel se fantasme chantant «Baby One More Time» et Artie (seul garçon de ces séquences) réinterprète «Stronger » 25 .

On note également un hommage à Michael Jackson («Michael» 3.11) ou encore à Whitney Houston ("Dance with Somebody » 3.17). Si Madonna ou Michael Jackson ont peut-être plus contribué à la subversion propre au queer que Britney Spears ou Whitney Houston ${ }^{26}$, tous sont des divas au sens où ils sont la persona

${ }^{21}$ Judith Butler, « From Interiority to Gender Performatives », Camp, Queer Aesthetics and the Performing Subject: A Reader, éd. Fabio Cleto, Édimbourg, Edimburgh University Press, 1999, p. 363-364 [p. 361-368].

${ }^{22}$ L'œuvre de Madonna comprend ses chansons et leurs paroles mais également ses clips vidéo ou encore ses diverses interventions publiques, qui, tous, participent du mythe Madonna tel que Georges-Claude Guilbert a pu l'étudier dans Le Mythe Madonna, Paris, Nouveau Monde Éditions, 2004.

23 Will Schuester explique à ses élèves : «Culturally Madonna's legacy transcends her music, because by and large, the subtexts of her songs are about being strong, independent and confident, no matter what your sex. But more than anything, Madonna's musical message is about equality. And that is something I think you guys need to work on. » (1.15)

24 Conformément au clip original de Britney Spears, auquel participe Madonna puisque la chanson est un duo, Brittany a le rôle de Britney Spears tandis que Santana endosse celui de Madonna.

${ }^{25}$ Artie est le seul garçon à figurer dans ces séquences fantasmées et, pourtant, il n’y a pas d'utilisation « queer » de cette réinterprétation puisqu'il est mis en scène dans un contexte classiquement masculin de joueur de football américain (on l'y voit sur le terrain, dans les vestiaires et à la salle de sport s'entraîner pour devenir plus fort et être accepté dans l'équipe malgré son handicap).

${ }^{26}$ On notera l'androgynie de la voix de Michael Jackson, certains de ses gestes tels le " crotch grabbing », justement repris par Madonna, la fluidité ou le relatif mystère de leur 
qu'ils ont construite et ne semblent pas avoir d'existence en dehors des oripeaux et du comportement de l'artiste qu'ils incarnent (comme s'ils n'existaient pas au naturel mais uniquement comme construction artificielle de leur persona d'artiste).

C'est cependant sur l'hommage rendu à Lady Gaga que l'on souhaite s'arrêter car elle répond encore mieux que tous les autres à l'ébauche de définition qui vient d'être donnée et illustre bien les rapports au déguisement et à l'imitation théorisés par Judith Butler. Chaque apparition publique de Lady Gaga est un véritable happening, qu'elle soit vêtue d'une robe en viande ${ }^{27}$ ou qu'elle sorte d'un ouf porté par ses danseurs, telle un extra-terrestre venant au monde devant les spectateurs ${ }^{28}$. Dans «Theatricality» (1.20), Kurt Hummel se rend compte avec effroi que la chorale rivale va faire un numéro de Lady Gaga, ce qui lui fait dire qu'ils vont utiliser la théâtralité la plus totale qui soit («They're doing Gaga [...] they're going with full-out theatricality. »). Puck (Noah Puckerman), personnage construit comme un sportif peu éclairé sur ces questions, lui répond : "What's up with this Gaga dude? He just, like, dresses weird right? Like Bowie? ». Cette remarque fonctionne sur deux plans. Il est tout d'abord intéressant que Puck se trompe sur le sexe de Lady Gaga («this Gaga dude »), ce qui multiplie encore les combinaisons possibles entre sexe et identités de genre chez cette artiste. Cependant, il la rapproche, en une interrogation finalement assez perspicace, de David Bowie, connu pour avoir été un très grand " gender-bender ${ }^{29}$ » à l'époque de son personnage de Ziggy Stardust et des beaux jours du club Studio 54 . Kurt, excédé, lui répond alors :

Lady Gaga is a woman. She's only the biggest pop act to come along in decades. She's boundary-pushing, the most theatrical performer of our generation and she changes her look faster than Brit3o changes sexual partners. (1.20)

La réponse de Kurt met l'accent sur l'absence de rigidité et la fluidité au coeur de l'identité de Lady Gaga. En la qualifiant de « boundarypushing ", il montre qu'elle ne s'arrête pas aux frontières fixes de l'identité classiquement définie (homme, femme, humain, etc.) et insiste sur le fait que tout chez elle est en même temps déguisement et essence («theatrical performer », « changes her look »).

sexualité, l'utilisation de costumes de scène tout à fait androgynes ou mêlant le masculin et le féminin.

${ }^{27}$ Lors des MTV Video Music Awards en 2010.

${ }^{28}$ Lors des Grammy Awards en 2011.

29 C'est-à-dire un artiste qui tord (littéralement) les catégories de genre en étant (dans l'exemple qui nous intéresse) un homme biologique jouant à adopter tour à tour des attributs de genre féminin ou masculin.

$3^{30}$ Leur camarade cheerleader et membre du " glee club » Brittany, qui change souvent de petit ami ou de petite amie puisqu'elle est bisexuelle. 
Après que leur professeur Will Schuester leur a annoncé que le thème imposé de la semaine était Lady Gaga, Finn, ainsi que tous les autres membres du "glee club » de sexe masculin (à l'exception de Kurt, point que l'on commentera plus tard), refusent de s'habiller comme Lady Gaga ou de chanter ses chansons et décident de se grimer comme les chanteurs du groupe KISS ${ }^{31}$. Le thème de la semaine n'est pas sans poser quelques difficultés aux élèves du «glee club » : alors que Tina et Kurt déambulent dans les couloirs du lycée habillés comme Lady Gaga dans certains de ses clips ou certaines de ses apparitions, ils sont violemment poussés contre les casiers par Dave Karofsky et Azimio. Kurt demande aux deux footballeurs de ne martyriser que lui et de laisser Tina tranquille ${ }^{32}$. Azimio lui répond : « Lately we haven't been able to tell the difference. We're not gaga for Gaga ». Le dialogue se poursuit ainsi :

DAVE. You dress all freaky and then rub it in everybody's faces. I don't want to look at it all day. It's weird. It makes my eyes tired. [...]

KURT. It's called being theatrical, we're showing off who we are. It's the same thing you do when you go to school with your football uniforms on. You're expressing yourself and we have every right to do the same.

(1.20)

Ce que montre cet échange opposant visuellement deux joueurs de football américain vêtus de leur « letter jacket » à Kurt et Tina " déguisés » en Lady Gaga, c'est qu'ils sont aussi déguisés les uns que les autres : en mettant cette veste, signe de leur appartenance à l'équipe de football, Karofsky et Azimio se déguisent en homme et illustrent l'idée que la masculinité (comme la féminité) est toujours déjà une performance.

Outre la question de la performance (qu'elle soit musicale ou entendue dans son sens queer) et le choix rarement innocent des artistes auquel il est rendu hommage dans Glee, il nous faut revenir sur l'aspect camp de la série. La première à avoir tenté de théoriser le camp est Susan Sontag avec « Notes on Camp ", datant de 1964. Ses réflexions ont longtemps fait autorité sur le sujet mais sont dorénavant remises en question, comme le montre le recueil édité par Fabio Cleto ${ }^{33}$. On retrouve cependant dans « Notes on Camp » quelques idées pouvant permettre de cerner cette notion aux contours flous. Ainsi,

${ }^{31}$ Ce n'est qu'à la fin de cet épisode que Finn accepte de se déguiser en Lady Gaga, une fois qu'il a admis que cela ne menace ni sa sexualité ni son identité d'homme.

$3^{2}$ À ce moment-là, Kurt adopte le comportement très classique du preux chevalier venant à l'aide de la demoiselle qu'il accompagne. On note d'ailleurs qu'il porte un costume argenté (en anglais, l'expression fait référence à un chevalier en armure brillante, " a knight in shining armour ») le rapprochant en même temps du genre masculin et du genre féminin (présence d'une jupe). Son geste, lui, relève du genre masculin de manière quasiment caricaturale.

33 Camp, Queer Aesthetics and the Performing Subject: A Reader, éd. Fabio Cleto, Édimbourg, Edimburgh University Press, 1999. 
Sontag écrit : " the essence of Camp is its essence of the unnatural: of artifice and exaggeration34. " Elle explique également que le camp est aussi une sensibilité, une manière de voir le monde particulière : " not in terms of beauty but in terms of the degree of artifice, of stylization ${ }^{35}$ », et va plus loin lorsqu'elle écrit ${ }^{36}$ : " Camp is a vision of the world in terms of style-but a particular kind of style. It is the love of the exaggerated, the "off", of things being-what-they-are-not ${ }^{37}$. »

Mark Booth, dans son article «Campe-toi!: On the Origins and Definitions of Camp ", propose une ébauche de définition claire et succincte : "To be camp is to present oneself as being committed to the marginal with a commitment greater than the marginal merits ${ }^{8}$. " À la suite de cette première définition, Booth précise ce qu'il entend par «the marginal ", à savoir "the traditionally feminine 39 ", «the trivial, the trashy, the kitsch and the not-terribly-good $4^{\circ}$. " La prévalence ainsi accordée par le camp à l'artificiel sur le naturel en fait le lieu privilégié d'une remise en question de l'ordre des priorités, montrant que tout est construit. Le naturel, ainsi que le lien soi-disant automatique et intrinsèque entre sexe et genre, sont donc disqualifiés.

Le personnage le plus camp de la série est sans aucun doute Kurt Hummel. Son prénom est une allusion à Kurt Von Trapp ${ }^{41}$, personnage de la comédie musicale The Sound of Music ${ }^{42}$, ce qui inscrit Kurt dans le sillon de Broadway et des comédies musicales, souvent associées au monde gay 43 . La manière dont Kurt voit le monde est éminemment camp. Il énonce parfois des règles telles que : " Every moment in your life is an opportunity for fashion » (1.3). Il mesure son humeur à l'aune de ses tenues : "I'm so depressed I've worn the same outfit twice » (1.21). Enfin, il subvertit les codes pré-établis des canons

\footnotetext{
34 Susan Sontag, « Notes on Camp », in Cleto, p. 53 [p. 53-65].

35 Sontag, p. 54

${ }^{36}$ Dans la huitième de ses « Notes on Camp ».

${ }^{37}$ Sontag, p. 56

$3^{8}$ Mark Booth, "Campe-toi!: On the Origins and Definitions of Camp », in Cleto, p. 69 [p. 66-79].

39 Ibid.

40 Booth, p. 70

41 Voir http://www.imdb.com/title/tt1327801/trivia?ref =tt trv trv (dernier accès le 8 août 2013).

${ }^{42}$ Cette comédie musicale date de 1959 et la musique en a été écrite par Richard Rodgers, les paroles par Oscar Hammerstein II, et le livret signé par Howard Lyndsay et Richard Crouse. C'est également le titre du film qui en est tiré, sorti sur les écrans en 1965.

43 Cette affinité entre les comédies musicales et le monde gay est expliquée ainsi par Pamela Robertson citant Robert Mast : «Perhaps there is something in two cultural clichés that make musicals and gay people especially suited to one another: musicals represent an extravagant and excessive frippery and gay people possess some special sensibility that finds a creative outlet in extravagance, excess, and frippery. " (Pamela Robertson, Guilty Pleasures: Feminist Camp from Mae West to Madonna, London, New-York, IB Tauris, 1996, p. 60). Le genre de la comédie musicale exprime donc une sensibilité camp et ceux qui se reconnaissent dans le camp trouvent là un moyen d'expression privilégié.
} 
de la mode, répondant «Fashion has no gender » à qui lui demande si le pull qu'il porte est un pull d'homme (2.1)

Si la mode (incarnation de l'artificiel, surtout dans la manière dont Kurt l'utilise) figure au panthéon de Kurt Hummel, les comédies musicales y sont en bonne place également. Dans 2.3, Kurt explique à son père Burt qu'il est plus important à ses yeux d'aller faire la queue pour acheter les DVD de la série Grey's Anatomy ou de se rendre à un " sing-along » de The Sound of Music 44 que de respecter la tradition du dîner en famille hebdomadaire. Ce rapport quasi religieux qu'entretient Kurt avec la musique, et celle de Broadway en particulier, se confirme lorsqu'il invoque " la colère de Sondheim » (" the wrath of Sondheim », 3.3), comme si Stephen Sondheim 45 était une divinité au courroux redoutable.

C'est un personnage qui permet alors une déconstruction particulièrement efficace des barrières entre les genres, les sexes, les activités, prélude à une fluidité queer que l'on n'aurait pas soupçonnée au premier abord. L'épisode qui nous intéresse particulièrement est intitulé « Preggers » (1.4) ${ }^{46}$. Il s'ouvre sur une scène dans laquelle Kurt, dans sa chambre située au sous-sol de la maison familiale, reproduit, en compagnie de Tina et de Brittany, la chorégraphie de Beyoncé et de ses danseuses dans le clip de la chanson "Single Ladies ». La scène est d'ailleurs filmée en couleurs puis en noir et blanc, comme dans le clip officiel. Kurt est clairement au cœur de la mise en scène, adoptant le rôle de Beyoncé tandis que ses camarades seraient les danseuses accompagnant l'artiste principale (voir figures 2 et 3).

$44 \mathrm{Il}$ va même jusqu’à dire que cet événement consistant à chanter les numéros musicaux de la comédie musicale en même temps qu'elle est projetée sur un écran est pour lui « sacré ». 45 Stephen Sondheim est un compositeur de très grandes comédies musicales, comme Sweeney Todd (1979) et c'est également le parolier de West Side Story (1957) ou encore de Gypsy (1959).

${ }^{4}$ Comme son titre l'indique, c'est l'épisode où l'on apprend la grossesse de l'apparemment si lisse Quinn Fabray, preuve finalement que tout n'est pas si « straight » qu'il y paraît au premier regard. 

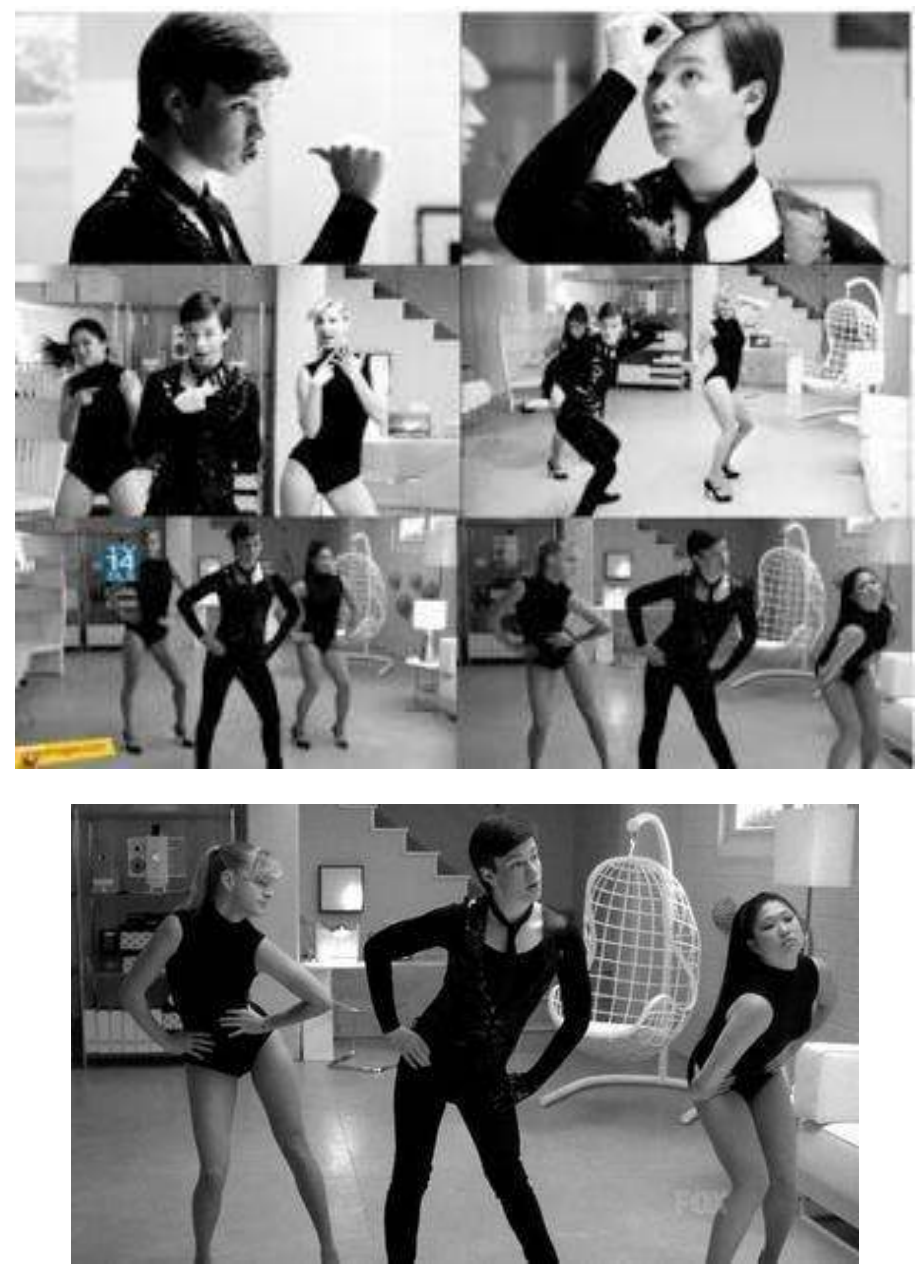

Fig. 2 et 3 : Kurt et ses danseuses sur la chanson Single Ladies de Beyoncé (1.4)

Le passage est interrompu, avec retour à la couleur, lorsque Burt, le père de Kurt, entre dans la chambre et arrête la musique. Kurt est manifestement surpris de voir son père déjà rentrer et ce dernier (vêtu d'une chemise de bûcheron et portant une casquette) lui dit d'un ton bourru que c'est l'heure de son émission, Deadliest Catch47, autant

47 Deadliest Catch (Discovery Channel, 2005-) est une émission à mi-chemin entre le documentaire et la télé-réalité, qui s'intéresse aux aventures d'équipages de marins- 
de signes contribuant à le marquer du sceau d'une masculinité un peu stéréotypée. Désireux d'expliquer la situation, manifestement embarrassante pour lui, Kurt répond à son père que ces deux jeunes filles sont ses petites amies et que ce sont des cheerleaders. L'une d'entre elles assure même à Burt que Kurt fait désormais partie de l'équipe de football américain, ce qui semble réjouir le père mais terrifier le fils. Après que Burt a quitté sa chambre, Kurt comprend qu'il va devoir essayer d'intégrer l'équipe de football, puisqu'il a promis à son père des billets pour son premier match. Ici, la performance est clairement reléguée à la marge, elle se fait dans le secret et semble comporter un élément très transgressif.

La deuxième scène se déroule à l'extérieur, sur le terrain de football. Finn (membre du « glee club » également) accepte de faire tenter sa chance à Kurt pour les essais de l'équipe. Kurt arrive sur le terrain alors que l'entraîneur fait des remontrances à un joueur (le botteur, celui qui tire au pied) dont les résultats depuis le début de l'année sont manifestement mauvais. Kurt se présente à l'entraîneur en ces mots : « Hi, I'm Kurt Hummel and I'll be auditioning for the role of kicker 48 ", manière dont on se présente à une audition de théâtre ou pour une comédie musicale, pas pour intégrer une équipe de sport. La déclaration de Kurt provoque d'ailleurs les rires à peine dissimulés des autres joueurs. Déjà, les codes se brouillent et les limites précédemment imperméables entre "glee club» et football se font plus floues. Avant de tirer, Kurt met en route le gros magnétophone qu'il a apporté et lance à nouveau la chanson «Single Ladies » de Beyoncé, au grand dam de l'entraîneur Ken Tanaka, qui marque clairement son atterrement: Kurt se livre alors à la même chorégraphie que dans sa chambre, mais cette fois-ci seul, vêtu de l'uniforme de football américain et entouré de footballeurs qui l'observent en riant franchement. Il danse pendant quelques secondes puis tape dans le ballon, qu'il envoie exactement où il faut avec autant de puissance que de précision, à la grande surprise des autres joueurs, qui en laissent tomber le casque qu'ils avaient à la main, et de l'entraîneur, qui l'accueille à bras ouverts dans l'équipe. La performance est ici moins isolée, puisqu'elle se fait sur un terrain qui est celui de la masculinité triomphante, en tenue de sportif. Kurt fait entrer la performance et une activité codée comme féminine (une danse, sur une chanson chantée par une femme, s'adressant aux femmes et parlant de femmes) dans un univers masculin, qu'elle lui permet qui plus est d'intégrer triomphalement.

pêcheurs dans la mer de Béring. C'est une émission qui vise particulièrement un public masculin par son format ainsi que son sujet, contribuant à parachever l'impression de masculinité déjà donnée par les vêtements du père de Kurt.

48 Au football américain, le " kicker » est le joueur chargé de frapper le ballon dans certaines phases de jeu. C'est l'équivalent du botteur (ou buteur) au rugby. 
Le troisième moment montre à quel point Kurt est arrivé à « queerer » l'univers de Glee : non content d'avoir rejoint l'équipe et d'avoir dansé sur une chanson chantée par une femme sur le terrain de football américain, il parvient à faire faire la même chose à ses coéquipiers, même aux plus récalcitrants. Cette scène a lieu lors d'un des tout premiers matchs de l'équipe de football du lycée auquel assiste notamment, comme promis, le père de Kurt. Au moment qui nous intéresse, l'équipe de McKinley est en train de perdre le match et Finn, en accord avec le reste de l'équipe et l'entraîneur, a décidé de tenter le tout pour le tout et de jouer sur l'élément de surprise. À son signal est donc diffusée dans les haut-parleurs « Single Ladies » et, cette fois-ci, c'est l'équipe tout entière qui se met à reproduire cette chorégraphie, dont certains pas sont même ébauchés par Ken Tanaka et quelques membres de l'équipe adverse, diversion ensuite utilisée par l'équipe de McKinley pour marquer un point, celui de l'égalisation puis de la victoire une fois que Kurt l'aura transformé.

On voit bien à travers la gradation de ces trois passages au cœur d'un même épisode que les frontières entre le sport et la danse ou le chant, entre le masculin et le féminin, deviennent de plus en plus poreuses, grâce notamment à Kurt, mais aussi et surtout à la performance (au sens anglais de représentation théâtrale) dansée qui mène ici à la performance (au sens d'exploit) sportive, montrant qu'elles ne sont bien évidemment pas antagonistes.

\section{La déconstruction des genres et de la sexualité}

Cependant, la déconstruction des barrières va plus loin encore, au travers de remarques tendant à montrer, épisode après épisode, que l'appartenance d'un sexe à un genre qui lui correspondrait comme naturellement est à repenser.

C'est sur le ton de la plaisanterie que sont faites les plus radicales de ces remarques, celles qui remettent en question la biologie même. Ainsi, Sue Sylvester, qui est habillée en survêtement en permanence, explique-t-elle qu'elle n'a aucun désir de procréer parce que, dit-elle : " I don't have the time, don't have the uterus » (1.6) et persiste en disant : «I don't menstruate » (2.2), comme si s'enlever ces attributs biologiques contribuait à la dépouiller de tout trait de caractère traditionnellement codé comme féminin telles la gentillesse, la douceur ou la bienveillance.

Sur le même thème, Kurt dit à ses amies Rachel et Mercedes, alors qu'ils sont tous les trois dans un lit à regarder une comédie romantique en mangeant du pop-corn : "Our periods don't come up until the end of the month » (3.12). Il joue ainsi sur ce que l'on appelle "l'effet gynécée ", qui voudrait que lorsque des jeunes filles ou des femmes passent beaucoup de temps ensemble, voire vivent ensemble, leurs cycles menstruels se synchronisent. En disant cela, Kurt marque 
son appartenance de fait au genre féminin à ce moment-là, mais pousser l'appartenance jusqu'à dire qu'il est doté d'attributs biologiques féminins a de quoi faire proliférer et déstabiliser les combinaisons sexe/genre.

Le jeu sur les attributs de genre n'est cependant jamais aussi efficace et subtil que lorsqu'il concerne les entraîneurs sportifs Sue Sylvester et Shannon Beiste (la remplaçante de Ken Tanaka à la tête de l'équipe de football). Shannon Beiste est une femme au gabarit peu courant et au physique relativement masculin au premier abord 49 , dont le personnage peut être qualifié de « gender-bending ». Si l'entraîneur précédent, Ken Tanaka, était globalement très opposé au " glee club ", et notamment au fait que «ses" footballeurs en fassent partie, Shannon Beiste - et ce n'est pas une coïncidence compte tenu de la position du personnage par rapport aux deux genres - y est très favorable et voit le rapprochement entre les deux activités comme le moyen de dégourdir ses athlètes et de régler les problèmes d'inimitié et de violence entre certaines brutes de l'équipe de football et leurs victimes. Les auteurs de la série s'amusent avec les attributs de genre de l'entraîneur de football américain, métier qui, traditionnellement, est plus souvent celui d'un homme que d'une femme : Sue ne dit-elle pas à Shannon qu'un entraîneur de football américain de sexe féminin - à l'instar d'un infirmier - est contre nature («A female football coach, like a male nurse, is a sin against nature » 2.1) ? Dans l'épisode $3 \cdot 3$, Shannon Beiste nous est montrée comme capable d'engloutir deux poulets rôtis à midi (faisant donc preuve d'un appétit vorace, plus souvent attaché au genre masculin qu'au genre féminin) et passant beaucoup de temps à la salle de sport à soulever de la fonte. Or dans l'épisode 3.5, elle s'exclame "I'm such a girl » face à son incapacité à retenir ses larmes dès qu'elle entend les élèves chanter le morceau « Tonight » tiré de la comédie musicale West Side Story. Ici, le terme de " girl », appliqué à une femme dans la fleur de l'âge, sert moins à faire référence à son sexe qu'à une certaine sensiblerie voire mièvrerie plutôt caractéristique d'une petite fille. Même si cela est péjoratif, c'est presque l'équivalent dans cet emploi du terme « gonzesse » en français. Dans ce même épisode pourtant, elle est montrée en train de souder des éléments du décor avec une joie certaine accompagnée de l'exclamation « God I love to weld $5^{\circ}$ », mais elle précise bien à un Artie

49 L'actrice Dot-Marie Jones a d'ailleurs joué dans le pilote de Pretty/Handsome dont l'histoire était centrée sur la transsexualité du personnage masculin principal. Notons également la prononciation du nom de famille du personnage (homophone de « beast») semblant mettre l'accent sur le fait que le personnage est situé en marge de la féminité ou de la masculinité classiques, dans un entre-deux présenté au premier abord comme quasi monstrueux.

$5^{0}$ « To weld » signifie « souder ». On pourrait arguer que, depuis Flashdance (réal. Adrian Lyne, 1983), une femme peut rester très féminine tout en étant en train de souder, mais la manière dont le personnage de Shannon Beiste se livre à cette activité la rapproche nettement plus des représentations classiques d'un homme qui bricole. 
essayant d'éviter tout faux pas que ce sont les hommes qu'elle aime («Guys, I like guys »). Cependant, le personnage est d'une timidité maladive et n'arrive pas à montrer ses sentiments à l'élu de son cœur, le recruteur Cooter Mankins. Elle ne sait lui déclarer sa flamme que d'une manière peu commune: "I'm telling you now if I had to benchpress a wildebeest just to prove to you how much you mean to me, I would do it. » (3.7). Prouver ses sentiments par un tel exploit de force physique n'est généralement pas l'apanage du genre féminin tel qu'il est classiquement envisagé51. En effet, ce que Shannon Beiste propose comme preuve de son amour est de soulever à l'aide la force de ses bras uniquement (allongée sur un banc de musculation, le sens exact de « to benchpress ») un gnou ( « a wildebeest »).

Ce sont finalement les joueurs de Shannon Beiste qui parviennent le mieux à la définir :

FINN. Coach Beiste we think you're awesome, and even though you're all hard and tough on the outside, it doesn't mean you're not the opposite on the inside.

SAM. Like a chocolate turtle ${ }^{2}$.

FINN. Totally. You're nougat-y. And we totally get that now. You're like...a mash-up. (2.6)

Cette notion de mash-up utilisée pour décrire Shannon Beiste est particulièrement importante dans l'économie de la série. Un mash-up est un genre musical hybride, qui consiste en l'association dans un même morceau de deux ou plusieurs titres existants, superposant généralement les parties musicales d'un morceau sur la musique d'un autre. De façon très parlante, ce mouvement de diagonale, de chiasme ou encore d'oblique caractéristique du mash-up (et du queer) est utilisé pour parler d'un personnage aux attributs de genre particulièrement fluides 53 .

Le personnage de Kurt illustre avec quelle subtilité ce sujet est traité dans Glee. Kurt s'associe régulièrement au groupe des filles. Alors que les garçons et les filles s'affrontent sur une performance musicale au sein du "glee club», Kurt dit: "Though I've been grouped with the boys my allegiances remain with you ladies » (1.6). Dans un autre épisode de la même saison (1.15), il déclare mériter le titre honorifique de « fille » ( « As an honorary girl I agree » 2.6). Cette dualité et cette fluidité de genre chez Kurt est confirmée lorsque, par

${ }^{51}$ La dernière saison ira même jusqu'à montrer Shannon Beiste battue par celui qui est devenu son époux. On l'y voit totalement soumise à cet homme violent malgré son gabarit physique : si l'habit ne fait pas le moine, le gabarit ne fait pas non plus automatiquement le genre (3.18 « Choke»).

${ }^{2}$ Il s'agit ici d'une référence à un type de friandise partageant les mêmes caractéristiques de mélange de texture.

53 Ce mouvement oblique caractéristique du queer est d'ailleurs également affirmé par Finn lorsqu'il accepte d'essayer de faire rentrer Kurt dans l'équipe de football américain : "The more crossover between glee club and football, the easier my life's gonna be » (1.4). 
exemple dans l'épisode 2.4, il chante une chanson de Victor/Victoria54 où il prend en charge le duo masculin/féminin à lui tout seul, se grimant et s'habillant d'un côté en homme, de l'autre en femme, passant indifféremment de l'un à l'autre durant sa prestation. Finalement, le seul moment où Kurt semble ne pas être à sa place, et même être déguisé, est lorsqu'il adopte les attributs de genre masculin, tant dans sa manière de s'habiller que de parler, et qu'il a une relation avec la cheerleader Brittany. Il devient alors une caricature de jeune homme hétérosexuel, voire une caricature de son propre père, et adopte les goûts de ce dernier, avec qui il va manger un hamburger en discutant de la musique de John Mellencamp. À propos de la chanson « Pink Houses », qui traite de la pauvreté en Amérique, Kurt dit : «It was very brave for a Midwesterner like himself to write a song about such bold interior design ", faisant alors ressortir sa vision camp du monde, plus intéressée par l'artifice de la mise en scène qu'est la décoration d'intérieur. C'est finalement après cet épisode peu concluant que Kurt s'acceptera pleinement et n'essaiera plus de changer qui il est pour plaire à son père 55 et répondre à une pression sociale diffuse. Notons, cependant, que même si Kurt est souvent associé au monde féminin, il est clairement établi que c'est un homme.

$\mathrm{Si}$ son sens du style est personnel et excentrique, abolissant parfois les limites entre les vêtements d'homme et ceux de femme, il ne s'habille pas en femme et refuse fermement de se travestir. Ainsi, lorsque le "glee club » cherche à mettre en scène le Rocky Horror Picture Show 56 (2.5 « Rocky Horror Glee Show ») et que Will Schuester lui demande de jouer le rôle de Frank-n-Furter57, Kurt refuse fermement: " There's no way I'm playing a transvestite in high heels and fishnets and wearing lipstick $5^{8}$ ". Il n'est pas non plus purement et simplement considéré comme une fille lorsqu'il s'agit d'élire le président des élèves et que Brittany se présente contre lui. Santana explique son refus - féministe - de voter pour Kurt en disant : « $\mathrm{He}$

\footnotetext{
54 Film musical réalisé par Blake Edwards en 1982

55 Alors qu'il partage un moment tendre avec Brittany, il laisse à son père un petit mot : " Do not enter under any circumstances, I'm making out with a girl », s'assurant par là que son père le voit bel et bien avec une jeune fille, pensant qu'ainsi leur relation père-fils hétérosexuel sera plus simple.

${ }^{5}$ Film musical réalisé par Jim Sharman en 1975 et adapté de la comédie musicale de Richard O’Brien datant de 1973.

57 Personnage qui est un homme travesti en femme: "a nice transvestite from Transylvania » (on cite les paroles de la comédie musicale).

$5^{8}$ C'est finalement Mercedes qui tiendra ce rôle, ce qui est beaucoup plus queer puisqu'elle devient alors une femme biologique jouant un homme biologique travesti en femme moment de jeu sur le genre et le sexe qui n'est pas sans rappeler le point de départ du film Transamerica (réal. Duncan Tucker, 2005) dans lequel une actrice (Felicity Huffman) joue le rôle d'un homme biologique en train de changer de sexe pour devenir une femme.
} 
looks like Jimmy Fallon's butch daughter59 but a vote for him would only empower another frank and beans » (3.3).

Cette attitude est à opposer à celle de Wade Adams, qui fait son apparition dans l'épisode 3.16: ce jeune homme fait partie de la chorale rivale (celle du lycée Carmel High, Vocal Adrenaline) et demande conseil à Kurt et Mercedes car il souhaite se produire sur scène habillé en femme (ce qu'il finit par faire malgré les recommandations de Kurt et de Mercedes) :

KURT. You can't do this. I admit that I've worn some flamboyant designer outfits, but I've never dressed up like a woman.

WADE. That's because you identify yourself as a man, I thought you of all people would understand. I'm wearing them ${ }^{60}$. (3.16)

Dans l'épisode 3.20, Wade Adams se produit donc à nouveau habillé en femme avec Vocal Adrenaline sous le nom d'Unique (son identité lorsqu'il est vêtu ainsi). Face au succès impressionnant de sa prestation, Sue Sylvester souhaite faire la même chose chez les New Directions :

SUE. He/She will trounce us at nationals. [...] It's time to fight fire with the flaming flames of additional flamey gay fire. [...]

KURT. Just because I'm gay doesn't mean I like to dress up like a woman. (3.20)

Kurt fait une différence nette entre affirmer la fluidité de son identité de genre (se déguiser en Lady Gaga n'est pas vraiment se déguiser en femme, l'artiste elle-même se plaçant au-delà du simple clivage homme/femme) d'un côté, et se déguiser tout bonnement en femme de l'autre. Son positionnement est donc plus queer que celui d'un homme qui s'habillerait en femme, puisque Kurt est dans le va-et-vient permanent entre le sexe auquel il appartient et les deux genres dont il peut tour à tour adopter les attributs (vestimentaires ou autres). S'il est une question sur laquelle Glee s'interroge, c'est donc bien celle de la sexualité et de l'hétéronormativité, en reprenant un certain nombre de clichés qu'elle fait voler en éclats et en faisant proliférer les combinaisons possibles de sexe/genre/sexualité.

Lorsque Shelby Corcoran (à la tête de la chorale Vocal Adrenaline) embrasse Will Schuester (1.14), elle lui demande : "Are you gay? 'Cause most of the show choir directors I make out with are

59 Jimmy Fallon est un animateur américain à la tête d'une émission satirique, Late Night with Jimmy Fallon, diffusée sur NBC depuis 2009. On note le jeu sur la combinaison genre/sexe : au lieu de dire que Kurt ressemble à un Jimmy Fallon un peu plus féminin, Santana dit qu'il ressemble à la fille lesbienne d'apparence masculine que l'animateur pourrait avoir, mais qu'il reste un homme (« frank and beans » étant une manière imagée et argotique de faire référence au sexe - au sens littéral du terme - masculin).

60 « Them » fait référence aux chaussures de femme que lui ont données Kurt et Mercedes pour l'inciter à se produire habillé en femme (ils ont en fait été encouragés par Sue Sylvester qui pensait ainsi ruiner toutes les chances de gagner de l'équipe rivale). 
gay. » À elle seule, cette phrase montre l'absence totale de rigidité dans sa sexualité : ces hommes qu'elle qualifie d'homosexuels l'ont embrassée mais cela ne la conduit pas à les envisager comme hétérosexuels pour autant. De la même manière, lorsque dans l'épisode 3.1, Kurt, alors engagé dans une relation homosexuelle avec Blaine Anderson, console son amie Rachel Berry, cette dernière lui dit : « You make me want to be your boyfriend right now ", montrant par là qu'elle s'identifierait au garçon dans cette potentielle relation amoureuse avec lui, tandis qu'elle l'associerait soit à un garçon homosexuel soit à une fille. La prolifération atteint des niveaux vertigineux et il y a tant de combinaisons possibles que la phrase de Rachel peut s’interpréter de multiples façons.

Ces différents points confirment bien que Glee propose un univers et des personnages queers. En effet, on aura noté le jeu (spatial mais également ludique, et parfois jubilatoire) mis en évidence par ces personnages appartenant à un sexe biologique dont ils adoptent ou pas les attributs de genre. C'est à notre sens cette fluidité qui permet à la série (sous des dehors légers de teen series acidulée) de proposer en fait une série militante distillant à ses téléspectateurs (principalement des adolescents potentiellement en proie à des questionnements sur leur sexualité ou leur orientation sexuelle) des messages progressistes et parfois libérateurs.

\section{Une série au militantisme queer}

La relation entre les personnages de Brittany et de Santana permet d'étudier l'infléchissement de la série au cours des saisons, évolution faisant de Glee une fiction de plus en plus militante sur les questions d'homosexualité, notamment chez les adolescents, mais également sur les questions de brimades et de harcèlement au lycée ${ }^{61}$.

La première mention d'une quelconque relation entre Santana et Brittany a lieu dans l'épisode 1.13, dans lequel la première explique que coucher ensemble n'est pas la même chose que sortir ensemble («Sex is not dating »), ce qui fait dire à la seconde que si c'était le cas alors Santana et elle sortiraient ensemble («If it were, Santana and I would be dating »). Au début, donc, ces deux jeunes filles sont décrites comme ayant des relations physiques sans pour autant que cela ait une quelconque incidence sur la manière dont elles envisagent leur sexualité : Brittany a des relations amoureuses avec à peu près tous les

61 Évoquons l'évolution du personnage de Dave Karofsky, la terreur des lycéens de McKinley et en particulier de Kurt Hummel, qu'il ne cesse de violenter. Dave se révèle être en fait homosexuel et incapable de l'accepter. Dans la troisième saison, on le voit enfin assumer son homosexualité sans pour autant qu'il se montre désireux de s'en ouvrir aux camarades de son nouveau lycée. Le jour où ces derniers découvrent sa sexualité et se mettent à le martyriser, il tente de se suicider - évolution radicale du personnage comme du message de la série. 
garçons du lycée, notamment les sportifs, tandis que Santana a une relation suivie avec Noah Puckerman. Au cours de la deuxième saison (dans l'épisode intitulé " Duets », 2.4), on voit d'ailleurs Santana et Brittany dans un moment intime, en train de s'embrasser, mais Santana prend bien garde de ne pas laisser cette activité définir sa sexualité en disant à Brittany :

I'm not making out with you because I'm in love with you and want to sing about making lady babies ${ }^{62}$. I'm only here because Puck's been in the slammer for about twelve hours now ${ }^{6} 3$ and I'm like a lizard: I need something warm beneath me or I can't digest my food. (2.4)

Pourtant, quelques épisodes plus tard (2.15), Santana accepte son désir d'une relation avec Brittany. Elle ne souhaite cependant pas se définir comme homosexuelle, expliquant ne pas être intéressée par les étiquettes ( "not interested in labels ») et conserver une attirance pour les garçons. Elle admet enfin, dans ce même épisode, être amoureuse de Brittany et souhaiter une relation amoureuse exclusive avec elle tout en continuant à refuser le terme de "lesbienne " et d'aller à des concerts des Indigo Girls ${ }^{64}$. À ce moment-là, Brittany entretient une relation relativement sérieuse avec Artie Abrams, qu'elle refuse de quitter pour Santana. Cette dernière, étonnée par la rigidité de son amie (le terme "stuck » signifiant ici « coincée ») s'exclame : "Wow, whoever thought that being fluid meant you could be so stuck? " C'est la première occurrence - mais pas la dernière - du terme «fluid » appliqué à la sexualité de ces adolescents, terme qui montre que l'on est peut-être dans un univers, ou à une période, où il est envisageable que la sexualité ne soit pas un paramètre fixe et figé mais bien changeant et variable selon les moments.

C'est dans l'épisode « Born This Way » (2.18, encore centré sur Lady Gaga et sa musique) que Santana commence à accepter sa sexualité. Lors de cet épisode, Will Schuester, en collaboration avec Emma Pillsbury, décide de demander à ses chanteurs d'interpréter l'hymne à la tolérance " Born this way » de Lady Gaga, vêtus d'un teeshirt blanc sur lequel ils auront fait imprimer ce qu'ils ont, ou ont eu, du mal à accepter ou souhaiteraient changer chez eux. Face aux résistances de Santana à accepter ses préférences sentimentales et sexuelles, Brittany décide de lui faire un tee-shirt sur lequel sera inscrit

\footnotetext{
${ }^{62}$ Le besoin d'une telle précision met en doute cette affirmation. Comme l'explique en effet Samuel Chambers, la meilleure manière de remettre en question son hétérosexualité est le besoin de l'affirmer : " The best way to call one's heterosexuality into question is to declare it, since if one is really straight one need never say so. » (Chambers, p. 123).

63 Rappelons ici que Santana est la petite amie officielle de Noah Puckerman (Puck), qui a commis un délit et vient d'être envoyé en maison de correction (" slammer » étant un terme familier pour faire référence à la prison).

${ }^{64}$ Les Indigo Girls sont un duo de rock folk féminin composé de femmes ouvertement homosexuelles, référence qui permet donc à Santana de mettre à distance l'étiquette « lesbienne».
} 
«Lesbian ». C'est sans compter sur le fait que Brittany est dépeinte dans la série comme relativement simple d'esprit - elle incarne le stéréotype de la blonde idiote - et c'est en fait un tee-shirt portant l'inscription "Lebanese » qu'elle offre à son amie. Ainsi, même au moment où Santana est censée accepter sa sexualité, la définition de cette sexualité reste inexacte, peu claire, ou en tout cas difficile à cerner précisément. Brittany, quant à elle, se déclare «bi-curious ", donc engagée dans l'exploration, la fluidité et la mouvance, et non prisonnière de l'étiquette figée d'une sexualité qui le serait tout autant $^{65}$. Ce n'est que dans l'épisode 3.7 que Santana semble avoir des certitudes sur sa sexualité et c'est dans cet épisode qu'elle décide de faire son coming-out, d'abord à elle-même puis à ses camarades, à sa mère et enfin à sa grand-mère. À l'occasion de cet épisode entièrement centré sur la question de l'éventuel coming-out de Santana, le personnage de Sue Sylvester 66 fait une remarque sur sa propre sexualité alors qu'elle se confie à son journal intime :

Why would someone assume I'm a friend of Ellen's ${ }^{67}$ just because I'm mannish and highly aggressive and have short hair and I only wear track suits and I coach a girl's sport and I married myself? It just doesn't make sense. The truth is journal I'm attracted to men. Sure, I can't stand watching them eat or talking to them, but when it comes to getting sexy; this gal's got a hole in her heart only a fella can fill. (3.7)

Le jeu permanent, particulièrement patent ici, entre ce que dit le personnage de Sue Sylvester et ce que les téléspectateurs savent de la vie personnelle de Jane Lynch ${ }^{68}$ permet de tricoter et de détricoter en même temps un discours sur la sexualité. Il est ici montré que rien ne

${ }^{65}$ Cela est confirmé dans l'épisode 3.19, lorsqu'elle fait le bilan de son année en disant : « I now realise I wasted an entire year belaboring the nuances of my fluid teen sexuality ». Il semble que Brittany déplore cette fluidité en disant qu'elle a " perdu une année entière à maudire les diverses nuances de sa sexualité adolescente caractérisée par la fluidité ». On peut penser que cette fluidité est décrite, du fait du champ lexical employé, comme peu positive. Cependant, on peut aussi se dire que la perte de temps réside dans la non acceptation automatique de cette fluidité : si elle avait été entendue dès le départ comme quelque chose de parfaitement " normal ", alors Brittany n'aurait pas perdu de temps et aurait simplement vécu cette sexualité fluide sans se poser de questions.

${ }^{66}$ Ce personnage est joué par Jane Lynch, lesbienne assumée, militante et mariée avec sa compagne, ce qui incite à prendre tout ce que dit Sue Sylvester avec du recul et du second degré.

${ }^{67}$ Il s'agit ici d'une référence à l'actrice/comique/animatrice Ellen deGeneres qui a fait son coming-out en direct à la télévision américaine chez Oprah Winfrey en 1997. C'est une lesbienne militante, mariée à l'actrice Portia de Rossi.

${ }^{68}$ Voir ce qu'écrit John Fiske sur l'intrusion du filmographique et du biographique dans la diégèse: «The actors and actresses who are cast to play hero/ines, villain/esses and supporting roles are real people whose appearance is already encoded by our social codes. But they are equally media people, who exist for the viewer intertextually, and whose meanings are also intertextual. They bring with them not only residues of the meanings of other roles that they have played, but also their meanings from other texts such as fan magazines, showbiz gossip columns, and television criticism. », Television culture, New York, Routledge, 1987, p. 5. 
peut assurer que quelqu'un est homosexuel ou hétérosexuel, en même temps qu'est mis en place un jeu avec les clichés de représentation de l'homosexualité, qui se trouvent confirmés en même temps qu'infirmés.

Tous ces éléments contribuent à des prises de position très progressistes sur la sexualité au sein d'un programme apparemment anodin, diffusé sur une chaîne qui n'est pas une chaîne du câble, et donc moins susceptible de donner une très grande liberté de parole : les personnages de Santana, de Brittany, de Kurt (et de Dave Karofsky) permettent de mettre en valeur la déconstruction de la sexualité comme donnée rigide de l'identité.

D'autres moments marquent une absence de corrélation entre la sexualité et des attributs de genre, notamment chez les jeunes hommes : être un homme ne signifie pas être un homme hétérosexuel. Lors de l'épisode 2.8 ( « Furt »), le père de Kurt et la mère de Finn, tous deux veufs depuis longtemps et follement épris l'un de l'autre, se marient. Finn fait alors un petit discours dans lequel il dit à son frère par alliance : « Kurt, no one else has shown me as much as you what it means to be a man ". Cette phrase montre bien que, non content de considérer qu'être homosexuel n'enlève rien à l'identité d'homme de Kurt, Finn fait de lui un modèle de masculinité. Au cours de la saison 3, le père de Kurt lui dira quelque chose d'approchant lorsque, fier de son fils qui vient de décrocher une audition pour une très prestigieuse école d'arts dramatiques, il prononce ces mots : "You and me, we made each other men ». Cette redéfinition de la masculinité autour d'attributs de genre remaniés culmine lorsque, dans l'épisode 3.22, Burt offre à son fils comme cadeau de fin d'année un spectacle lors duquel il danse sur scène sur la chanson de Beyoncé "Single Ladies ", comme son fils dans l'épisode précédemment évoqué.

\section{«Queerer » la définition de la famille}

Finalement, outre une redéfinition de la masculinité et de la féminité tout autant qu'une remise en question de l'hétéronormativité, ce qu'apporte Glee, de manière plus ou moins ouverte, ce sont des éléments queers redéfinissant ce que signifie le terme de famille ${ }^{69}$.

Ainsi, lorsque le « glee club » perd la compétition nationale de chant choral parce que les disputes au sein du groupe ont été nombreuses, Brittany explique que ce genre de disputes arrive dans toutes les familles :

SANTANA. Yeah, well, this is a club. This is not a family.

${ }^{69}$ Terme chargé de sens s'il en est en ce moment, particulièrement aux États-Unis comme en France avec les discussions sur le mariage gay, l'adoption et l'accès à la PMA pour les couples homosexuels, qui remettent en question l'image traditionnelle et la définition classique voire biblique de la famille. 
BRITTANY. Okay well, family is a place where everyone loves you no matter what, and they accept you for who you are. I know I'm going to be a bridesmaid at Mike and Tina's wedding, and I'm gonna be anxiously waiting, just like everyone else to see if their babies are Asian too. When they find an operation to make Artie's legs work again I'm going to be there for his first steps. I love them. I love everyone in glee club. And I get to spend another year with everyone I love, so, I'm good. SANTANA. What about you and I?

BRITTANY. I love you Santana. I love you more than I've ever loved anyone else in this world. All I know about you and I is that, and because of that, I think anything's possible. (2.22)

Cette redéfinition du concept de famille au sein du « glee club » est rendue possible par l'aspect queer de cet espace caractérisé par l'entre-deux (ni parfaitement masculin, ni féminin par exemple), comme le montre ce que dit Kurt à la fin de la troisième saison, alors qu'il s'apprête à quitter le lycée et, donc, ses camarades :

I wanted to mostly thank the men in the room who have truly inspired me and never saw me for the things that made us different. You only saw me for the ways that we're the same. Because in this room [the show choir room] it doesn't matter if you're gay or straight, what matters is that we're friends. (3.22)

Voilà donc l'espace du "glee club » investi d'un pouvoir magique de dissolution de l'identité, préalable nécessaire à la reconstruction de la personnalité de ses membres autour du concept de reprise musicale, de performance.

Cette prise de position va même plus loin lorsque Santana et Kurt, à des moments différents, parlent à Dave Karofsky de l'avenir qu'ils imaginent pour lui. Santana, dans l'épisode 2.14, comprend par tout un faisceau d'indices que Dave est homosexuel mais bien loin de se l'avouer, de l'assumer et, plus encore, de le vivre ouvertement. Elle lui dit alors :

You're what we call a late-in-life gay. You're going to stay in the closet, get married, get drunk to have relations with your wife, have a couple of kids, maybe become a state senator or a deacon and then get caught in the men's room tapping your foot with some page ${ }^{70}$. And I accept that about you. (2.14)

Au cours de la troisième saison (3.14), après que Dave Karofsky a été harcelé par les garçons de son nouveau lycée et a tenté de se suicider, Kurt va le voir à l'hôpital et lui tient un discours bien différent: il lui dépeint un avenir pas si lointain où il pourra être

${ }^{\circ 0}$ Il s'agit ici d'une expression signifiant littéralement " se faire attraper dans les toilettes pour hommes en train de taper du pied avec un jeune huissier ». Il semblerait que taper du pied dans une cabine de toilettes publiques (et attendre qu'un autre homme montre son intérêt pour la proposition en faisant de même) soit une manière pour certains hommes homosexuels de trouver un partenaire du même sexe pour une relation de courte durée. 
ouvertement homosexuel, agent de sportifs et père d'un enfant, dans la ville de son choix.

Ces deux perspectives sont bien différentes et Ryan Murphy comme Brad Falchuk semblent ainsi montrer les deux voies qui s'ouvrent à nos sociétés : d'un côté, un avenir peu enviable où les homosexuels vivent une vie faite de honte et de mensonge ; de l'autre, une vie très mainstream où l'on peut être homosexuel et heureux, jouissant des mêmes droits que les autres, même en travaillant dans un milieu traditionnellement peu «gay-friendly » ${ }^{71}$. «Tomorrow ${ }^{72}$ ", semblent nous chanter Murphy et Falchuk dans Glee, accompagnés de manière quasi chorale par les nombreux acteurs homosexuels, mariés et pères de famille qu'ils font intervenir en guest-stars dans la série (Neil Patrick Harris ${ }^{73}$, Matt Bomer ${ }^{74}$ ou encore Ricky Martin75).

Grâce à la notion de reprise, Glee est une série marquée par le renversement. En effet, les mêmes éléments qui semblaient contenir le comportement de ses protagonistes dans un cadre très rigide, normatif et hétéronormé sont en fait subvertis et mis à mal par la reprise, musicale notamment, permettant à la performance théâtrale de se transformer en performance queer.

Ce renversement est particulièrement visible dans l'usage promotionnel fait des fameux «slushies » évoqués plusieurs fois dans cette étude. Au départ, on l'a vu, lancer un «slushie » au visage d'un autre lycéen est une manière de l'humilier en tachant ses vêtements (le "slushie » est souvent de couleur vive, rouge notamment), en le mouillant et en s'assurant qu'il va être recouvert d'une espèce de sirop poisseux le reste de la journée. Le geste n'a rien d'anodin, tant concrètement que symboliquement (on l'a vu également avec la " mise à mort » par «slushie » d'Artie). Pourtant, le lancer de «slushie » fait partie intégrante de la campagne promotionnelle de la série intitulée " Gleek out ». On y voit en effet tous les membres du " glee club » (ceux-là mêmes qui sont régulièrement malmenés) lancer un "slushie » coloré (bleu ou rouge) en direction de celui qui regarde l'image (voir figure 4).

\footnotetext{
${ }^{71}$ Est-ce là un manifeste quasi politique quand on connaît la date de diffusion de l'épisode 3.14 ( 21 février 2012) et le contexte des élections présidentielles, qui voient un vrai durcissement de la droite américaine sur les questions sociales et les « culture wars »?

72 « Tomorrow » est une chanson phare de la comédie musicale Annie (musique de Charles Strouse et paroles de Martin Charnin, 1977) dans laquelle la jeune orpheline Annie chante avec optimisme sa foi dans un avenir radieux.

73 « Dream On » (1.19)

74 « Big Brother » (3.15)

75 « The Spanish Teacher » (3.12)
} 


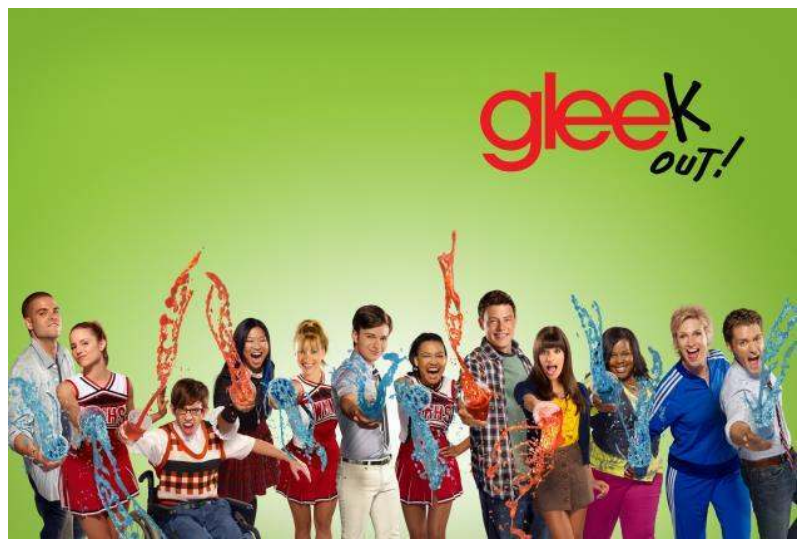

Fig. 4 : Une des affiches de la campagne promotionnelle « Gleek out ».

La reprise est ici particulièrement jubilatoire grâce à plusieurs éléments visuels. On remarque tout d'abord que tous les personnages sourient en faisant ce geste. Ensuite, les couleurs de cette série d'affiches sont particulièrement vives et acidulées, connotant quelque chose de très joyeux. Enfin, on ne sait pas à qui est destiné ce " slushie " : est-ce une vengeance lancée au visage de ceux qui étaient leurs bourreaux ? Est-ce une manière de reprendre à leur tour un geste humiliant et d'en faire (grâce à la joie et la jubilation qui émane des photos) quelque chose d'amusant et de divertissant?

Il en va de même pour l'autre partie de la campagne promotionnelle « Gleek out » où l'on voit tous les protagonistes mimer avec leur pouce et leur index le « L » de « loser » sur leurs fronts (voir figure 5), geste habituellement fait par les élèves les plus populaires comme insulte à l'intention de ceux qu'ils considèrent comme des « perdants », des « nazes ». 


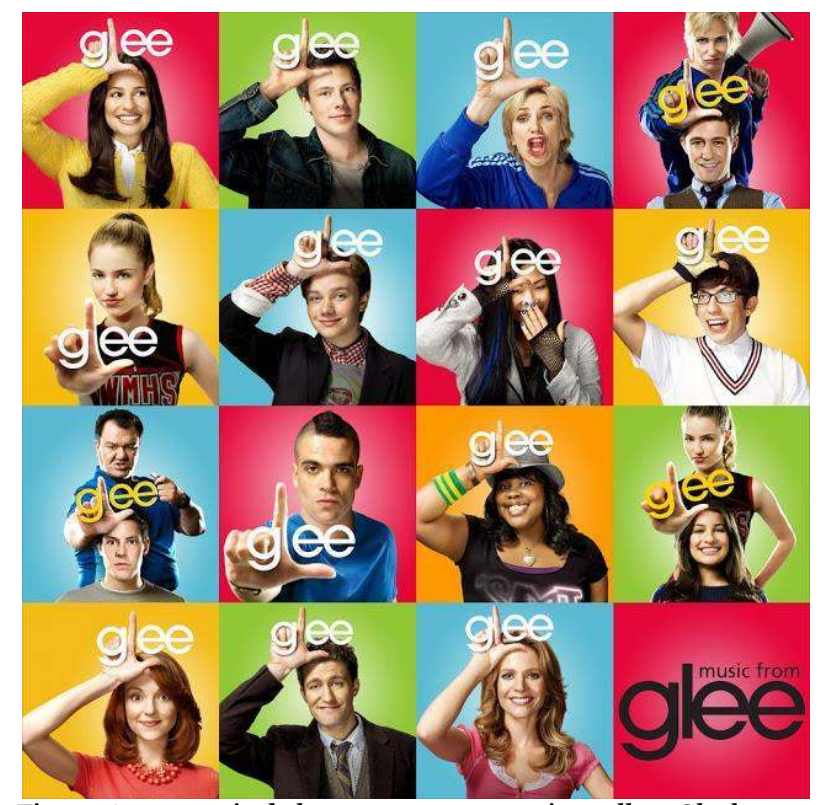

Fig. 5 : Autre partie de la campagne promotionnelle « Gleek out »

Que les membres du «glee club» fassent de même sur des affiches aux couleurs pop, où ils apparaissent particulièrement enjoués, montre à nouveau comment ils renversent les codes et les vident de leur valeur par la multiplication inflationniste de leur représentation. Voilà donc une campagne promotionnelle qui, à elle seule, résume l'aspect jubilatoire et subversif de la reprise dans Glee.

\section{Bibliographie}

BuTLER Judith, Gender trouble, New-York, Londres, Routledge, 1990.

BUTLER Judith, «From Interiority to Gender Performatives» in Camp, Queer Aesthetics and the performing Subject: A Reader, éd. Fabio Cleto, Édimbourg, Edimburgh University Press, 1999, p. 361-368.

Bоотн Mark, «Campe-toi ! On the origins and definitions of Camp », in Camp, Queer Aesthetics and the performing Subject: A Reader, éd. Fabio Cleto, Édimbourg, Edimburgh University Press, 1999, p.66-79.

Chabrol Marguerite, «Glee: Theatricality! », Positif, n607, 2011, p. 103-105. 
Chambers Samuel A., The Queer Politics of Television, Londres, NewYork, IB Tauris, 2009.

FISKE John, Television culture, New York, Routledge, 1987.

Guilbert Georges-Claude, Le Mythe Madonna. Paris, Nouveau Monde Éditions, 2004.

KIMMEL Michael, « Masculinity as Homophobia » in Reconstructing Gender: A Multicultural Anthology, éd. Estelle Disch, Boston, McGraw Hill, 2008, p. 103-109.

Robertson Pamela, Guilty Pleasures: Feminist Camp from Mae West to Madonna, London, New York, IB Tauris, 1996.

SonTAg Susan, " Notes on Camp » in Camp, Queer Aesthetics and the Performing Subject: A Reader, éd. Fabio Cleto, Édimbourg, Edinburgh University Press, 1999, p. 53-65.

\section{L'auteure}

Virginie Marcucci est professeur d'anglais en classes préparatoires. Elle a soutenu sa thèse sous la direction de Georges-Claude Guilbert sur le féminisme dans la série Desperate Housewives et vient de publier Desperate Housewives, un plaisir coupable? aux Presses Universitaires de France. Ses domaines de recherche de prédilection sont les gender et queer studies, le camp et le féminisme, notamment dans les séries télévisées (ou sitcoms) américaines. 